\title{
New formulation of metallic materials constitutive models by an energy approach
}

\author{
B. Saade ${ }^{\dagger}$, A. Gavrus ${ }^{*}$, J. Bodgi $^{\dagger}$ and N. Bejjani ${ }^{\dagger}$ \\ ${ }^{\dagger}$ Département Mathématiques, Laboratoire Mathématiques et Applications \\ Université Saint-Joseph de Beyrouth \\ Campus des Sciences et Technologies, Mar Roukos - Dekwaneh, Liban \\ Laboratoire de Génie Civil et de Génie Mécanique LGCGM (EA 3913) \\ Institut National des Sciences Appliquées de Rennes \\ 35700 Rennes, France \\ Email: bechara.saade@insa-rennes.fr \\ * Laboratoire de Génie Civil et de Génie Mécanique LGCGM (EA 3913) \\ Institut National des Sciences Appliquées de Rennes \\ 35700 Rennes, France \\ e-mail: adinel.gavrus@insa-rennes.fr, web page: https://agavrus.perso.insa-rennes.fr/ \\ ${ }^{\dagger}$ Département Mathématiques, Laboratoire Mathématiques et Applications \\ Université Saint-Joseph de Beyrouth \\ Campus des Sciences et Technologies, Mar Roukos - Dekwaneh, Liban. \\ Email: joanna.bodgi@usj.edu.lb \\ ${ }^{\dagger}$ Département Mathématiques, Laboratoire Mathématiques et Applications \\ Université Saint-Joseph de Beyrouth \\ Campus des Sciences et technologies, Mar Roukos - Dekwaneh, Liban. \\ Email: nadine.bejjani@net.usj.edu.lb
}

\begin{abstract}
In a classical way the Cauchy stress tensor $\sigma$ is expressed in function of instantaneous internal variables as the strain, the strain rate and the temperature. If good predictions of the response of materials are obtained under quasi-static loadings, inaccurate estimations are finding for some severe dynamic conditions where high plastic strain, high strain rate or temperature gradients and microstructures changes occur during the plastic flow. To describe the metals work hardening under specific loadings, the traditional laws reduce the influence of the material deformation history only through the cumulated plastic strain $\bar{\varepsilon}$ defined from the time integral of the generalized strain rate. From a thermodynamic point of view the material stress is rather related to the dissipated plastic strain energy responsible for any change in the material thermomechanical state. This work propose then a new formalism to define a material constitutive equation from an equivalent stress $\bar{\sigma}$ expressed as a function of the dissipated plastic deformation energy: $\bar{\sigma}=f(W), W=\int \bar{\sigma} d \bar{\varepsilon}$ with $g\left(W, \frac{d W}{d \bar{t}}\right)=0$. This new approach takes into account the microstructure changes which govern a lot of the physical mechanisms responsible for plastic flows: work-hardening, dynamic softening and phase transformation. This formulation remains valid for large paths of material deformation and for all the spectrum of loading conditions: from static, transient until rapid dynamic ones. Variational energetic approaches were first mentioned by R. HILL [1] indicating that the work-hardening phenomenon depends on the plastic strain energy. Recently a new formalism introduced by M. YOSHINO et al. [2] define for a first time a named reference stress as a function of plastic strain energy. From a thermodynamics point of view a reliable energy approach must be based on the principle that any system finds its time evolution by minimizing the losses energies and minimising the generated entropy [3]. One of the main objectives of this work is to find in an energetic framework the proposed general constitutive laws [4]: $\bar{\sigma}=\sigma_{h}(\bar{\varepsilon}) \sigma(\dot{\bar{\varepsilon}}, T)(1-\psi)+\sigma_{s} \sigma(\dot{\bar{\varepsilon}}, T) \psi$ with $\sigma(\dot{\bar{\varepsilon}} . T)=G(\dot{\bar{\varepsilon}}) H(T)$ and $\mathrm{d} \psi / d \bar{\varepsilon}=R(\bar{\varepsilon})(1-\psi)$, where $\psi$ is the material fraction undergoing dynamic softening (recovery, recrystallization or phase transformation). Firstly will be finding the energetic form of the usual hardening formulations of $\sigma_{h}(\varepsilon)$ proving the equivalency between the new formulation and the traditional ones i.e: $\sigma_{h}=H(\bar{\varepsilon}) \Leftrightarrow \sigma_{h}=f(W)$ with $W=\int \sigma_{h} d \bar{\varepsilon}$. The obtained new constitutive model will be discussed comparing to a numerical implementation on the incremental elastic-plastic Prandtl-Reuss equations resolution.
\end{abstract}

\section{REFERENCES}

[1] R. Hill, The Mathematical theory of plasticity, Oxford, The Clarendon Press, (1950).

[2] M. Yoshino et T. Shirakashi, Flow-stress equation including effects of strain-rate and temperature history, Int. $J$. Mech. Sci., 39 (12) :1345-1362 (1997).

[3] A. Bejan, Advanced Engineering Thermodynamics, 2nd ed. Wiley, New York, (1997).

[4] A. Gavrus, Constitutive equation for description of metallic materials behavior during static and dynamic loadings taking into account important gradients of plastic deformation, Key Engineering Materials, 504-506:697702 (2012). 